\title{
Novel six-nucleotide deletion in the hepatic fructose-1,6-bisphosphate aldolase gene in a patient with hereditary fructose intolerance and enzyme structure-function implications
}

\author{
R ita Santamaria ${ }^{1}$, L uigi V itagliano ${ }^{2}$, Sonia Tamasi $^{1}$, Paola I $z_{2}{ }^{1}$, L ucia Zancan ${ }^{3}$, \\ A driana Zagari ${ }^{2}$ and Francesco Salvatore ${ }^{1}$ \\ ${ }^{1}$ D ipartimento di B iochimica e B iotecnologie M ediche, CE IN GE -B iotecnologie A vanzate, U niversità di Napoli \\ Federico II, Naples, Italy \\ ${ }^{2}$ Centro di Studio di Biocristallografia, CNR and Dipartimento di Chimica, Università di Napoli Federico II, Naples, \\ Italy \\ ${ }^{3}$ D ipartimento di Pediatria, Università di Padova, Padua, Italy
}

\begin{abstract}
Hereditary fructose intolerance $(\mathrm{HFI})$ is an autosomal recessive human disease that results from the deficiency of the hepatic aldolase isoenzyme. A ffected individuals will succumb to the disease unless it is readily diagnosed and fructose eliminated from the diet. Simple and noninvasive diagnosis is now possible by direct D NA analysis that scans for known and unknown mutations. U sing a combination of several PCR -based methods (restriction enzyme digestion, allele specific oligonucleotide hybridisation, single strand conformation analysis and direct sequencing) we identified a novel six-nucleotide deletion in exon 6 of the aldolase $B$ gene ( $\Delta 6$ ex6) that leads to the elimination of two amino acid residues (Leu182 and Val183) leaving the message inframe. The three-dimensional structural alterations induced in the enzyme by $\Delta 6$ ex6 have been elucidated by molecular graphics analysis using the crystal structure of the rabbit muscle aldolase as reference model. These studies showed that the elimination of Leu182 and Val183 perturbs the correct orientation of adjacent catalytic residues such as Lys146 and G lu187.
\end{abstract}

Keywords: inborn errors of metabolism; hereditary fructose intolerance; human aldolase B; mutation-causing enzyme alterations; molecular graphics analysis

\section{Introduction}

A Idolase is a homotetrameric enzyme that catalyses the reversible aldol cleavage of fructose 1,6-bisphosphate

Correspondence: Professor Francesco Salvatore, Dipartimento di Biochimica e Biotecnologie Mediche, CEINGEBiotecnologie A vanzate, U niversità di Napoli 'Federico II', Via S Pansini 5, I-80131 Naples, I taly. Tel: + (39) 0817463135; Fax: + (39) 081 7463650; E-mail: salvator@unina.it

R eceived 6 A ugust 1998; revised 230 ctober 1998; accepted 11 November 1998
(FBP) and fructose 1-phosphate (F 1P). There are three aldolase isoenzymes in mammals: A (muscle), B (liver) and $\mathrm{C}$ (brain). ${ }^{1} \mathrm{H}$ ereditary fructose intolerance $(\mathrm{HFI})$, inherited in an autosomal recessive fashion with an estimated frequency of 1 in 20000 live births, is an inborn error of carbohydrate metabolism caused by a deficiency of aldolase $\mathrm{B}$. The clinical manifestations of $\mathrm{HFI}$, following ingestion of fructose, sucrose or sorbitol, consist in abdominal pain, vomiting, and such metabolic disturbances as hypoglycaemia that may be fatal. 
Symptoms usually regress once fructose is totally excluded from the diet. ${ }^{2}$

A Idolase $B$ is encoded by a single gene and more than 20 gene mutations have been described. The most frequent in Europe are three missense mutations, $A$ 149P and A 174D in exon 5, and N 334K in exon 9. ${ }^{3-6}$

Crystallographic studies of human aldolase $B$ at high resolution (J A Littlechild, personal communication, 1998) are in progress, although only low-resolution $\left(3.8 A^{\circ}\right)$ diffracting crystals have been published so far. ${ }^{7}$ D ifferently, the 3-D structure of rabbit aldolase A has been extensively studied at high resolution (about $\left.2 A^{\circ}\right)^{8-12}$ and recently a study of the rabbit aldolase $A$ crystal structure containing dihydroxyacetone phosphate casts light on the molecular structure in the bound and unbound states. ${ }^{12}$

We have found a novel mutation in an I talian $\mathrm{HFI}$ patient consisting of a deletion of six nucleotides in exon 6 of the aldolase $B$ gene, which alters the structure of the hepatic aldolase, thus resulting in a barely active enzyme.

\section{Materials and Methods}

\section{Patient and Aldolase B Activity}

The subject is a 6-year-old child. Hereditary fructose intolerance was suspected at 5 months of age on the basis of typical clinical symptoms and elevated serum aspartate transaminase levels. The clinical diagnosis was confirmed by greatly reduced aldolase B activity in liver biopsy. The fructose-1-phosphate (F1P) aldolase activity was $0.056 \mathrm{U} / \mathrm{g}$ (normal value 1.82-3.40 U/g), the fructose-1,6-bisphosphate (FBP) aldolase activity was $0.41 \mathrm{U} / \mathrm{g}$ (normal value $2.07-3.79 \mathrm{U} / \mathrm{g}$ ), and the substrate activity ratio FBP/FIP was 7.3 (normal value 1.0-1.2); lastly, the fructose-1,6-bisphosphatase (FBPase) activity was $1.29 \mathrm{U} / \mathrm{g}$ (normal value $2.73-4.98 \mathrm{U} / \mathrm{g}$ )

The patient had liver failure, characterised by hepatomegaly, steatosis and fibrosis of hepatic cells, ascertained after needle biopsy. In the first two years of life, the weight gain was within the 25 percentile and height increase within the 50 percentile with respect to controls of the same age. At present, his weight is within the 75 percentile and height within the 50 percentile, compared with age-matched controls

\section{DNA Analysis}

G enomic D NA was isolated from nucleated peripheral blood cells according to a standard procedure. ${ }^{13}$ The conditions used for DNA amplification (PCR) and for allele-specific oligonucleotide hybridisation (A SO) analysis for the most frequent mutations (A 149P, A 174D and N334K) were as previously described. ${ }^{14,15} \mathrm{~A}$ mplified DNA products containing the coding regions of the aldolase $B$ gene were examined by single strand conformation polymorphism (SSCP) analysis carried out at $4^{\circ} \mathrm{C}$ on a $10 \%$ non-denaturing polyacrylamide gel in the presence of $5 \%$ glycerol, as previously described. ${ }^{15}$
The amplified fragments were directly sequenced in both directions using the Sequenase PCR Product Sequencing $K$ it (USB, Cleveland, O hio, USA), according to the manufacturer's instructions. PCR-amplified genomic fragments containing exon 6 were digested with Avall and $R$ sal endonucleases (B oehringer, M annheim, G ermany), according to the manufacturer's recommendations, and the products were analysed by electrophoresis on a $12 \%$ polyacrylamide gel and visualised after ethidium bromide staining.

\section{Molecular Graphic Analysis}

Modelling of human aldolase $B$ was carried out using the structure of rabbit aldolase $A$ at $1.9 \mathrm{~A}$ resolution (Protein $D$ ata $B$ ank, code $1 A D O)$. The sequence identity between the two isozymes is $69 \%$, with neither insertions nor deletions. In particular, the sequence identity is $85 \%$ in the encompassing region of the deletion site (aa 159-191). A ll the catalytic residues are conserved except from $A$ sn41 which is replaced by a lysine in rabbit aldolase $A$.

$\mathrm{R}$ abbit aldolase $\mathrm{A}$ structural motifs were analysed using the programme PROM OTIF. ${ }^{16}$ B ecause the mutated fragment is far from the subunit interfaces in the tetramer, we confined our analysis to a single subunit that is known to possess catalytic activity, albeit at a level lower than the tetrameric structure.

The search for loops connecting the $\alpha$-helix D (Ser159A sn180) and the $\beta$-strand 5 (Val183-L eu191) was performed using a database of high resolution structures implemented in the programme $\mathrm{O}^{17}$ (Figure 3 was drawn using $\mathrm{MOL}$ SCRIPT ${ }^{18}$ and RA STE R-3D. ${ }^{19}$ )

\section{Results and Discussion}

\section{DNA Mutation Analysis}

Using PCR and ASO hybridisation techniques we found the patient was heterozygous for mutation N334K in exon 9. SSCP analysis of the entire coding region revealed a shift in the electrophoretic mobility only in exon 6 (Figure 1A ). Direct sequencing analysis showed a six-nucleotide deletion (from 10072 to 10077), corresponding to codons 182 and 183, leaving the downstream coding region inframe (Figure 1B). This new mutation, assigned the trivial name $\Delta 6 \mathrm{ex} 6$, and the systematic name g10072-10077del, ${ }^{20}$ creates a new A va II site (G/G(AT)CC) and abolishes an Rsa I site (GT/A C) (see Figure 2).

R estriction digestion analysis (Figure 2 ) revealed that the patient inherited the $\Delta 6 \mathrm{ex} 6$ mutation from the father. The N $334 \mathrm{~K}$ mutation in exon 9 , as revealed by A SO hybridisation (data not shown), was carried by the mother, heterozygous for this mutation. Direct sequencing of both parents DNA confirmed that the mutation followed Mendelian inheritance (data not shown). Restriction analysis of $\Delta 6 \mathrm{ex} 6$ in the proband's 5 -month-old brother showed normal patterns (see Figure 2). 
A

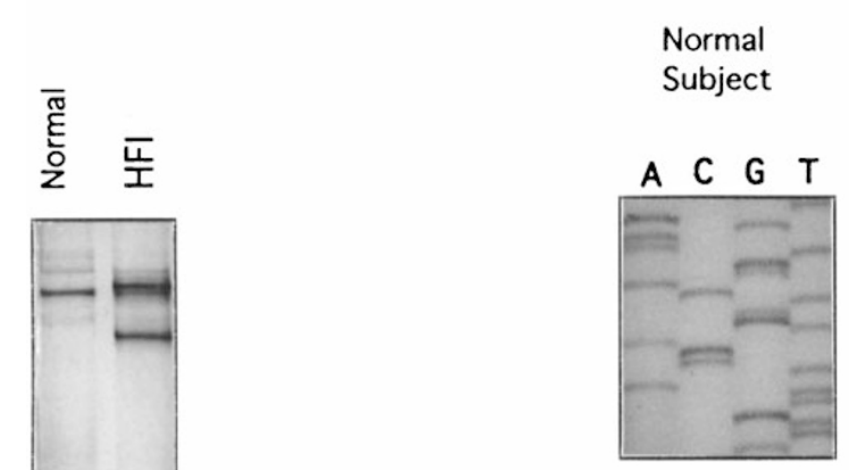

B

$\mathrm{HFI}$

Subject

Normal sequence

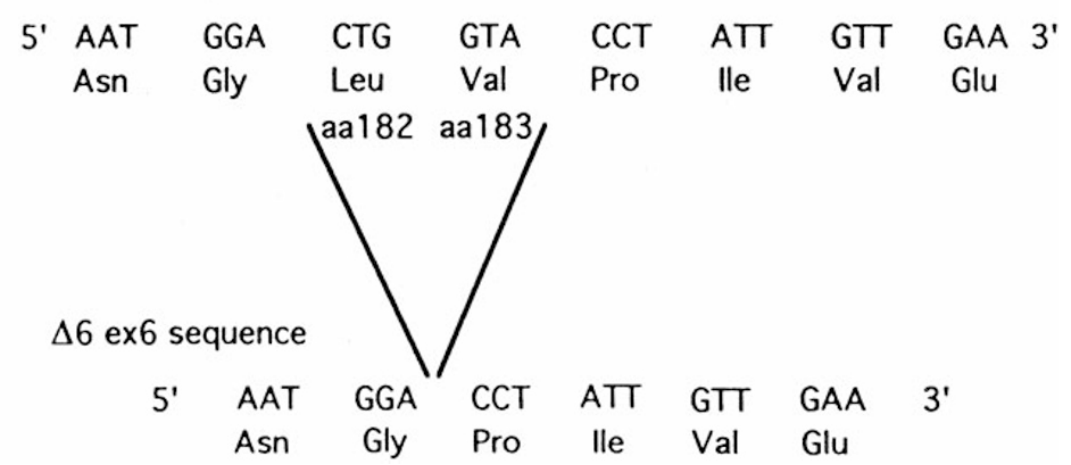

Figure 1 D etection of the $\triangle 6$ ex 6 mutation in the human aldolase B gene. A: Single strand conformation polymorphism analysis of exon 6 amplified DNA in normal and HFI subjects. B: Direct sequencing of exon 6 amplified DNA from a normal subject and the proband. The deletion site is marked by an arrow; normal and mutated sequences and codon translation are indicated; the deleted region in the $\mathrm{HFI}$ subject includes the two amino acids, L eu 182 and Val 183

The newly identified mutation ( $\Delta 6 \mathrm{ex} 6)$ was not a polymorphism since it was absent from the normal population, as determined by restriction analysis of 60 chromosomes from 30 unrelated individuals.

The mutation described in this paper consists in the deletion of two amino acids which are highly conserved: Leu182, which is replaced in some vertebrates only by isoleucine, and Val183, which is present in all class I aldolases. The two amino acids are located near the catalytic site of the enzyme. Therefore, their elimination could cause inactivation of aldolase $B$. This is strongly supported by a negligible F IP aldolase B activity in the patient's liver. The other mutation N 344K carried by our patient has previously been found in the homozygous state associated with $\mathrm{HFI}^{4}$ Consequently, it appears that the $\Delta 6$ ex 6 mutation is critical for aldolase B function. As verified in 60 normal chromosomes, mutation $\Delta 6 \mathrm{ex} 6$ is not present in the normal population, except in the proband's father in the heterozygous state. Therefore, this mutation together with mutation N334K, is the cause of HFI in the proband.

\section{Enzyme Structure-Function Implications}

The aldolase molecular architecture corresponds to an $(\alpha / \beta)_{8}$ barrel with the active site in a deep pocket at the $\mathrm{C}$-terminal end. Consequently $\alpha$-helices are identified by the letters from $A$ to $H$ and $\beta$-strands by numbers from 1 to 8 (Figure $3 A$ ). The pocket is lined primarily by charged amino acids that interact with the substrate. A mong others, Lys146 and Glu187 are directly bound to D HA P, the product of the catalytic reaction. ${ }^{12}$ These residues are supposed to interact with the C1-phosphate moiety and to act as proton donors/ acceptors during the catalytic reaction which proceeds through a Schiff base intermediate involving proton transfers. ${ }^{12}$

In aldolases, L eu182 belongs to a loop connecting the $\alpha$-helix D with the $\beta$-strand 5 (Figure 3B) and Val 183 is 
Ava II

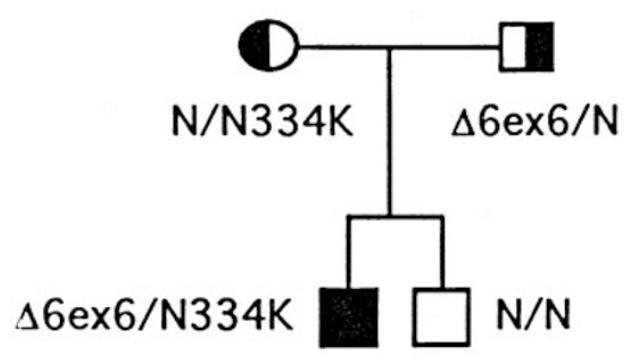

U

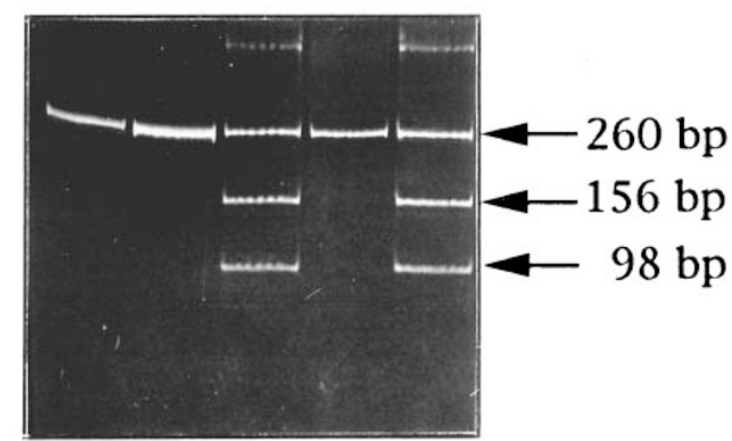

Rsa I

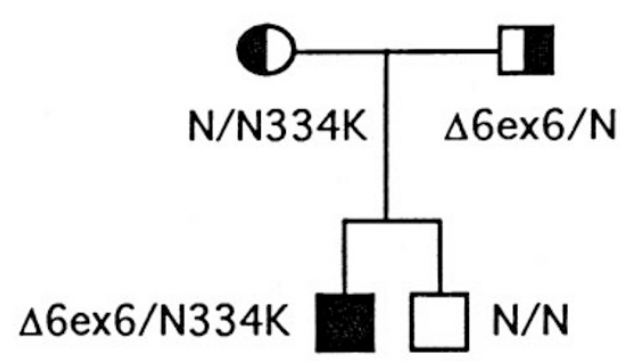

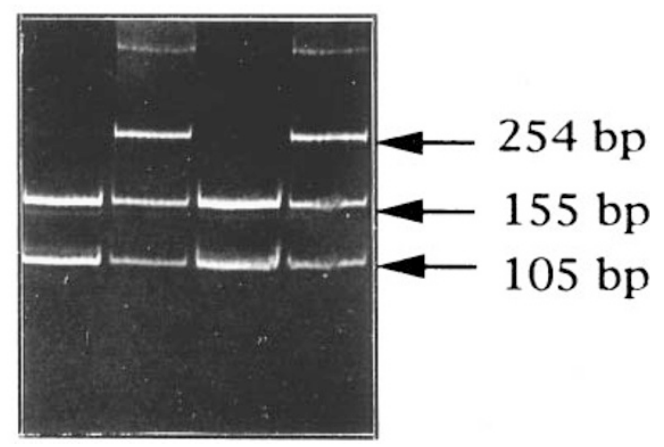

Figure 2 Segregation of the $\Delta 6$ ex 6 mutation in the proband's family by restriction enzyme digestion analysis of exon 6 amplified DNA. The lanes of the electrophoregrams correspond vertically to the subjects indicated in the pedigrees of the family, which are reported in the upper part of the Figure together with the corresponding genotypes; $U$ indicates the undigested 260-bp PCR product. $L$ eft: digestion with the restriction enzyme Ava II. The normal fragment of $260 \mathrm{bp}$ is present in all subjects, and in the patient and in his father the fragments of $156 \mathrm{bp}$ and $98 \mathrm{bp}$ are present because of the mutation. Right: the pattern after digestion with the restriction enzyme Rsa I. The mother and the brother of the proband show the normal fragments of $155 \mathrm{bp}$ and $105 \mathrm{bp}$; the patient and his father show both the normal fragments ( $155 \mathrm{bp}$ and $105 \mathrm{bp}$ ), and the fragment of $254 \mathrm{bp}$ (present because of the mutation) indicating a heterozygous state for this mutation

the first residue of strand 5. Val183 links A sp143 and A la145, which are located in the near $\beta$-strand 4, through two hydrogen bonds. Therefore, the $\mathrm{N}$-terminal ends of strands 4 and 5 , which are two adjacent staves of the $\beta$-barrel, are maintained close to each other by these hydrogen bonds. To explore the structural alterations resulting from the deletion, we carried out a systematic search for closure $\alpha$-helix $D$ with the $\beta$-strand 5, after omitting residues L eu182 and Val 183. Various possible closures were found with satisfactory stereochemistry, indicating that the mutated enzyme should be able to fold. However, to rebuild this connection, a variation of the backbone conformation of at least three residues (amino acids 179-181) is required. The mutation produces, therefore, the unwinding of the C-cap amino acids $179-180$ of the $\alpha$-helix and the weakening of the contacts between $\mathrm{N}$-ends of strands 4 and 5 . This might severely alter the helix/strand connection. Furthermore, it is noteworthy that the catalytic residues Lys146 and Glu187 are located in $\beta$-strand 4 and 5 respectively and are not far from the deletion site. This proximity may induce small local changes to side chains of Lys146 and Glu187 and these variations may explain the remarkable reduction in enzyme activity towards both substrates $(0.056 \mathrm{U} / \mathrm{g}$ for FIP and 0.41 for FBP). Indeed, the substrate activity ratio FBP/FIP of 7.3 , which was observed in our patient's liver biopsy, is markedly higher than normal values. The more pronounced activity reduction towards F IP is consistent with a local rearrangement of Lys146 and Glu187 in the mutated enzyme. These residues are located in the active site region that recognises and interacts with the $\mathrm{C}-1$ phosphate moiety; ${ }^{11}$ even small movements of their side chains of Lys146 and Glu187 can weaken these favourable interactions.

In conclusion, the severe clinical symptoms of $\mathrm{HFI}$ observed in our proband support, albeit indirectly, the 


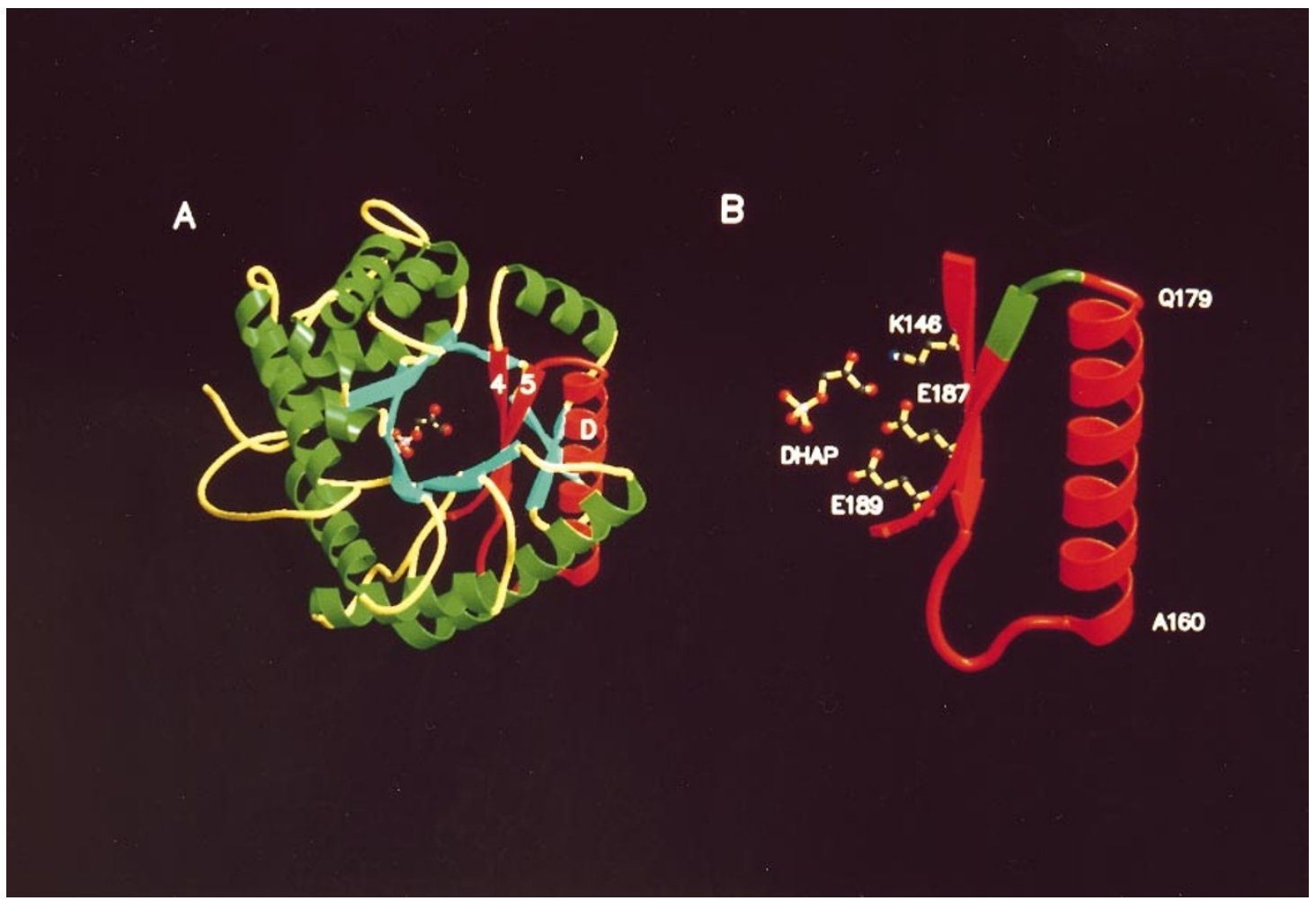

Figure 3 Three-dimensional structure of rabbit aldolase A with $\alpha$-helices and $\beta$-strands drawn as a coiled ribbon and flattened arrows, respectively. A : O verall $(\alpha / \beta)_{8}$ barrel of a subunit of rabbit aldolase $\mathrm{A}: \alpha$-helices and $\beta$-strands are shown in green and blue, respectively, other connecting regions are in yellow. The $\mathrm{N}$-terminal $\alpha$-helix $\mathrm{A}$ has been omitted, for clarity. The region corresponding to the $\alpha$-helix D and the $\beta$-strands 4 and 5 , where the latter includes the deleted residues 182-183, is shown in red. D H AP bound to the enzyme is shown in the centre. $\mathbf{B}$ : D etail of panel $\mathbf{A}$, showing the region of the deletion site. R esidues 182-183 are shown in green. Side chains of Lys146 (strand 4), Glu187 and Glu189 (strand5), that belong to the active site, are also shown. Note the DHAP molecule at the active site

role ascribed to Lys146 and G lu187 in agreement with findings emerging from the crystal structures. ${ }^{8,12}$ Therefore, the analysis of altered protein conformation by molecular graphic modelling contributes to the understanding of the proper functioning at the enzyme active site.

\section{Disease Mutation Frequency and Genetic Screening}

In the 28Italian patients studied by us the most frequent H FI mutations A 149P and A 174D account for $66 \%$ of mutant alleles, whereas the other $\mathrm{HFI}$ mutations account for the remaining 34\% (see Table 1 ). Interestingly, the heterogeneity of the mutation increases going from northern to southern European countries, as is the case of other hereditary diseases, eg cystic fibrosis. ${ }^{21}$ This higher heterogeneity of southern versus northern Europe accurately reflects the migrations typical of the Mediterranean basin.
Table 1 A llele frequency distribution of $\mathrm{HFI}$ mutations in I taly and North E uropean countries

\begin{tabular}{|c|c|c|}
\hline Mutation & Italy $(\%)$ & North E uropean countries $(\%)$ \\
\hline A 149P & 30.0 & 67.0 \\
\hline A 174D & 36.0 & 12.5 \\
\hline N 334K & 3.6 & 8.0 \\
\hline Other mutations & 30.4 & 12.5 \\
\hline
\end{tabular}

${ }^{\mathrm{a} D}$ ata from our case-mix on 56 alleles; ${ }^{\mathrm{b}}$ data from $\mathrm{Cox}^{5}$

The experimental approach to the diagnosis of $\mathrm{HFI}$, based on DNA analysis, can replace the intravenous fructose tolerance challenge test and invasive liver biopsy. Therefore this approach is useful for the prevention and therapy of this hereditary disease.

\section{Acknowledgements}

The authors are indebted to N B lom (M ontreal, Canada) for making available the coordinates of rabbit muscle aldolase $A$ prior to deposition. The assistance of $G$ Sorrentino for 
Figure 3 is acknowledged. We thank Jean Gilder for editing the text. This work was supported by grants from : M U RST (PRIN, 1997); CNR (Target Projects 'Biotechnology' and 'Biologia Strutturale'), R ome; R egione Campania 'R icerca Sanitaria Finalizzata' and 'A gen-Sud', R ome, Italy.

\section{References}

1 Salvatore F, Izzo P, Paolella G : A Idolase gene and protein families: structure, expression and pathophysiology. In: F. Blasi (ed). Human Genes and Diseases, Horizon in Biochemistry and Biophysics, vol. 8, John Wiley $\&$ Sons, L td, 1986; 611-665.

2 Gitzelmann R, Steinmann B, Van den Berghe G: D isorders of fructose metabolism. In: Scriver CR, Beaudet $A L$, Sly WS, Walle D (eds). The M etabolic and M olecular B ases of Inherited Disease, 7th edn. McG raw-H ill: New York, 1994,pp 905-934.

3 A li M, R ellos P, Cox TM : Hereditary fructose intolerance. J Med G enet 1998; 35: 353-365.

4 Cross NCP, Stojanov LM, Cox TM : A new aldolase B variant, N $334 \mathrm{~K}$, is a common cause of hereditary fructose intolerance in Yugoslavia. Nucleic Acids Res 1990; 18: 1925.

5 Cox TM : A Idolase B and fructose intolerance. FASE B J 1994; 8: 62-71.

6 Tolan DR: M olecular basis of hereditary fructose intolerance: mutations and polymorphisms in the human aldolase B gene. Hum M utat 1995; 6: 210-218.

7 Dalby A, Rawas A, Watson HC, Littlechild JA: Crystallisation and preliminary $\mathrm{X}$-ray diffraction studies on human liver aldolase. Protein Peptide Lett 1996; 3: 207-212.

8 Gamblin SJ, Cooper B, M illar JR, D avies J G, Littlechild JA, Watson HC: The crystal structure of human muscle aldolase at 3.0 A resolution. FEBS Lett 1990; 262: 282-286.

9 Gamblin SJ, Davies GJ, G rimes J M, Jackson R M, Littlechild JA, Watson HC: A ctivity and specificity of human aldolases. J Mol Biol 1991; 219: 573-576.
10 Littlechild JA, Watson H C: A data-based reaction mechanism for type I fructose bisphosphate aldolase. TIBS 1993; 18: 36-39.

11 Sygusch J, Beaudry D, A llaire M : Molecular architecture of rabbit skeletal muscle aldolase at $2.7 \mathrm{~A}$ resolution. Proc Natl A cad Sci USA 1987; 85: 9133-9137.

12 Blom N, Sygusch J: Product binding and role of the C-terminal region in class I D-fructose 1,6-bisphosphate aldolase. N at Struct Biol 1997; 4: 36-39.

13 M iller SA, D ykes DD, Polesky HF: A simple salting out procedure for extracting DNA from human nucleated cells. N ucleic A cids Res 1988; 16: 1215.

14 Santamaria R, Scarano M I, E sposito G, C hiandetti L, I zzo $\mathrm{P}$, Salvatore $\mathrm{F}$ : The molecular basis of hereditary fructose intolerance in Italian children. Eur J Clin Chem Clin B iochem 1993; 31: 675-678.

15 Santamaria R, Tamasi S, Del Piano G et al: Molecular basis of hereditary fructose intolerance in I taly: identification of two novel mutations in the aldolase $B$ gene. J M ed G enet 1996; 33: 786-788.

16 H utchinson EG, Thornton J M : PR O M OTIF : a program to identify and analyze structural motifs in proteins. Protein Science 1996; 5: 212-220.

17 Jones TA, Zou JY, Cowan SW, K jeldgaard M: Improved methods for building protein models in electron-density maps and the location of errors in these models. A cta Crystallogr 1991; A 47: 110-119.

$18 \mathrm{~K}$ raulis $\mathrm{PJ}$ : MOLSCRIPT: a program to produce both detailed and schematic plots of protein structures. J A ppl Crystallogr 1991; 24: 946-950.

19 M errit $E$, M urphy $M$ : R A STER 3D version 2.0: a program for photorealistic molecular graphics. A cta Crystallogr 1994; D 50: 869-879.

20 Beutler E, McKusick VA, Motulsky AG, Scriver CR $\mathrm{H}$ utchinson $\mathrm{F}$ : $M$ utation nomenclature: nicknames, systematic names, and unique identifiers. $\mathrm{H}$ um M utat 1996; 8: 203-206.

21 Castaldo G, Rippa E, Sebastio G et al: Molecular epidemiology of cystic fibrosis mutations and haplotypes in southern Italy evaluated with an improved semiautomated robotic procedure. J Med Genet 1996; 33 475-479. 